\title{
Cataract Surgery in Behçet's Disease Patients One Week after Infliximab Administration
}

\author{
Tsuneaki Handa Hinako Tsunekawa Masahiro Zako \\ Department of Ophthalmology, Aichi Medical University, Nagakute, Japan
}

\section{Key Words}

Infliximab · Behçet's disease · Cataract surgery · Infection · Tumor necrosis factor-a

\begin{abstract}
Purpose: To describe two patients with Behçet's disease who underwent cataract surgery one week after infliximab administration.

Methods: Patients received preoperative antibiotic therapy with $0.5 \%$ levofloxacin eye drops and $500 \mathrm{mg}$ levofloxacin oral tablets, followed by cataract surgery one week after infliximab administration. We observed ocular findings following cataract surgery, phacoemulsification-aspiration, and posterior chamber intraocular lens implantation.

Results: There were no intraoperative complications. Neither ocular inflammatory attacks nor infectious complications were found in the operated eyes of both patients during follow-up.
\end{abstract}

Conclusion: Administration of infliximab one week before cataract surgery is safe and effective for patients with Behçet's disease.

\section{Introduction}

Infliximab, a humanized antibody against tumor necrosis factor- $\alpha$ (TNF- $\alpha$ ), reduces uveitis attacks in patients with Behçet's disease (BD) [1,2]. Anti-TNF- $\alpha$ therapy increases the risk of infections due to the systemic blockade of TNF- $\alpha$ [3]. BD patients often require intraocular surgery for the treatment of accompanying cataract, glaucoma, or other vitreoretinal problems. However, it is uncertain whether administration of infliximab suppresses surgically induced ocular inflammation or increases the rate of surgeryassociated infections in BD patients. Furthermore, the most appropriate timing of intraocular surgery after infliximab treatment is unknown. Here, we report two patients with BD who successfully underwent cataract surgery one week after the last preoperative infliximab administration without suffering any postoperative complications. 


\section{Case Reports}

Infliximab therapy at $5 \mathrm{mg} / \mathrm{kg}$ was intravenously administered at 0 and 6 weeks, and every 8 weeks thereafter. Both patients underwent cataract surgery one week after the final preoperative infliximab administration. Patients received the following preoperative antibiotic therapy: $0.5 \%$ levofloxacin eye drops (Santen Pharmaceutical Company, Osaka, Japan) and $500 \mathrm{mg}$ levofloxacin oral tablets (Daiichi Pharmaceutical Company, Tokyo, Japan) [4]. Levofloxacin eye drops were administered beginning one week before surgery and were administered four times per day for a month. One levofloxacin tablet per day was administered in the morning starting the day before surgery and continuing for one week. As standard postoperative treatment, $0.1 \%$ betamethasone sodium phosphate eye drops (Shionogi and Co., Ltd., Osaka, Japan) were administered after surgery four times per day for three months. There were no intraoperative complications.

\section{Case 1}

The patient was a 41-year-old man with a 16-year history of BD. He had a nuclear and cortical cataract in the left eye, and underwent phacoemulsification-aspiration and posterior chamber intraocular lens (PEA+IOL) implantation with microincision $(2.4 \mathrm{~mm})$ of the scleral-corneal tunnel in August 2010. His best-corrected visual acuity (BCVA) improved from 0.03 to 0.05 . The impaired postsurgical visual acuity was due to severe optic nerve atrophy.

\section{Case 2}

The patient was a 37-year-old man with a one-year history of BD. He had a nuclear and cortical cataract in the right eye and underwent PEA+IOL implantation with microincision $(2.4 \mathrm{~mm})$ of the scleral-corneal tunnel in July 2010. His BCVA improved from counting fingers to 1.2.

Neither ocular inflammatory attacks nor infectious complications were found in the operated eyes of either patient during postoperative follow-up.

\section{Discussion}

Results from this small case series demonstrate that cataract surgery one week after administration of infliximab is safe and effective for patients with BD. No adverse effects, such as infection, occurred.

To our knowledge, there are only two published reports describing cataract surgery under infliximab therapy in patients with $\mathrm{BD}[5,6]$. In these cases, all patients underwent surgery about half way through an eight-week interval between doses of infliximab, and no patient showed postoperative uveitis or infection. As the serum levels of infliximab significantly correlate with its effectiveness in preventing recurrent episodes of uveitis [7], this midpoint between infliximab doses may be appropriate in determining timing of surgery based on the risk of infection associated with drug concentration. However, the trend of serum levels after infliximab administration showed strong interindividual variability associated with patient-specific pharmacokinetics, in particular the formation of anti-infliximab antibodies [8]. Here we performed cataract surgery one week after the last infliximab administration, an anticipated timing of reduced influence by individual pharmacokinetics, without suffering any postoperative complications including infection. In this shorter period after infliximab administration, serum concentration of infliximab is expected to remain high, and thus we believe it to be safer than surgery performed four weeks after infliximab administration in terms of preventing surgery-associated intraocular inflammation in $\mathrm{BD}$ patients. 
The risk of ocular infection in patients with BD on anti-TNF- $\alpha$ therapy has not been investigated in detail, but an increased risk in patients with rheumatoid arthritis was recently demonstrated. Epidemiological data on Japanese patients with rheumatoid arthritis revealed that the use of TNF- $\alpha$ inhibitors is a significant independent risk factor for serious infection when compared to treatment with nonbiological disease-modifying antirheumatic drugs (relative risk 2.37, 95\% confidence interval (CI) $1.11-5.05, \mathrm{p}=0.026$ ) [9]. Similarly, multivariate logistic regression analysis revealed that anti-TNF- $\alpha$ therapy was a significant risk factor for acute surgical site infection (odds ratio 5.69, 95\% CI 2.07$15.61, \mathrm{p}=0.0007$ ) and that infliximab increased the risk of surgical site infection (odds ratio $9.80,95 \%$ CI $2.41-39.82, \mathrm{p}=0.001$ ) [10]. A shorter period after infliximab administration correlates with higher serum drug concentration, and thus we should be prudent in terms of surgery-associated intraocular infection in BD patients.

\section{Disclosure Statement}

Proprietary interest: none. Grants and funds received in support of the study: none.

\section{References}

1 Sfikakis PP, Theodossiadis PG, Katsiari CG, Kaklamanis P, Markomichelakis NN: Effect of infliximab on sightthreatening panuveitis in Behçet's disease. Lancet 2001;358:295-296.

2 Ohno S, Nakamura S, Hori S, Shimakawa M, Kawashima H, Mochizuki M, Sugita S, Ueno S, Yoshizaki K, Inaba G: Efficacy, safety, and pharmacokinetics of multiple administration of infliximab in Behçet's disease with refractory uveoretinitis. J Rheumatol 2004;31:1362-1368.

3 Ellerin T, Rubin RH, Weinblatt ME: Infections and anti-tumor necrosis factor alpha therapy. Arthritis Rheum 2003;48:3013-3022.

4 Ishida M, Kataoka T, Niwa K, Iwaki M, Zako M: Efficient penetration into aqueous humor by administration of oral and topical levofloxacin. J Ocul Pharmacol Ther, in press.

5 Noda E, Yamanishi S, Shiraishi A, Ohashi Y: Cataract surgery under infliximab therapy in a patient with Behçet's disease. J Ocul Pharmacol Ther 2009;25:467-470.

6 Sakai T, Kanetaka A, Noro T, Tsuneoka H: Intraocular surgery in patients receiving infliximab therapy for Behçet disease. Jpn J Ophthalmol 2010;54:360-361.

7 Sugita S, Yamada Y, Mochizuki M: Relationship between serum infliximab levels and acute uveitis attacks in patients with Behcet disease. Br J Ophthalmol 2011;95:549-552.

8 Svenson M, Geborek P, Saxne T, Bendtzen K: Monitoring patients treated with anti-TNF-alpha biopharmaceuticals: assessing serum infliximab and anti-infliximab antibodies. Rheumatology (Oxford) 2007;46:1828-1834

9 Komano Y, Tanaka M, Nanki T, Koike R, Sakai R, Kameda H, Nakajima A, Saito K, Takeno M, Atsumi T, Tohma S, Ito S, Tamura N, Fujii T, Sawada T, Ida H, Hashiramoto A, Koike T, Ishigatsubo Y, Eguchi K, Tanaka Y, Takeuchi T, Miyasaka N, Harigai M: Incidence and risk factors for serious infection in patients with rheumatoid arthritis treated with tumor necrosis factor inhibitors: a report from the Registry of Japanese Rheumatoid Arthritis Patients for Longterm Safety. J Rheumatol, in press.

10 Momohara S, Kawakami K, Iwamoto T, Yano K, Sakuma Y, Hiroshima R, Imamura H, Masuda I, Tokita A, Ikari K: Prosthetic joint infection after total hip or knee arthroplasty in rheumatoid arthritis patients treated with nonbiologic and biologic disease-modifying antirheumatic drugs. Mod Rheumatol, in press. 